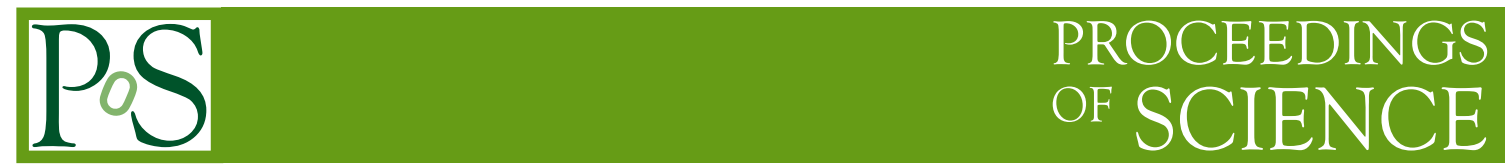

\title{
WIMPless Dark Matter: Models and Signatures
}

\author{
Jason Kumar* \\ University of Hawaii \\ E-mail: jkumar@hawaii.edu
}

We consider experimental signatures of WIMPless dark matter. We focus on models where the WIMPless dark matter candidate is a Majorana fermion, and dark matter scattering is predominantly spin-dependent. These models can be probed by IceCube/DeepCore, which can potentially find $3 \sigma$ evidence with $\sim 5$ years of data.

35th International Conference of High Energy Physics - ICHEP2010,

July 22-28, 2010

Paris France

${ }^{*}$ Speaker. 


\section{Introduction}

WIMPless dark matter [1] is a robust and versatile scenario in which the dark matter candidate is a hidden sector particle whose mass is at the hidden sector soft-SUSY breaking scale. Although the dark matter candidate can be either a boson or fermion and its mass can vary over a wide range (10 MeV $\lesssim \mathrm{m}_{\mathrm{X}} \lesssim 10 \mathrm{TeV}$ ), it automatically has the approximately correct relic density to match cosmological observations. The versatility of this scenario implies that specific models can have a wide variety of possible signatures, detectable using many different dark matter search strategies.

WIMPless dark matter signatures depend on whether the candidate is a boson or fermion. If the WIMPless candidate (the lightest particle stabilized by a hidden sector symmetry) is a scalar, this WIMPless candidate can have a much large spin-independent scattering cross-section $\left(\sigma_{\mathrm{SI}}\right)$ than one would expect from neutralino WIMPs. Models of this type have been studied [2] as a way of explaining the recent data of the DAMA, CoGeNT and CRESST experiments [3]. Signatures of these models can be studied at detectors such as the Tevatron, the LHC, Super-Kamiokande, and liquid scintillator neutrino detectors [4].

In these proceedings we focus on a different signature which can be found in a complementary set of models: spin-dependent scattering. Several detectors exist which are sensitive to the spindependent scattering cross-section $\left(\sigma_{\mathrm{SD}}\right)$. But bounds on $\sigma_{\mathrm{SI}}$ are much tighter than those on $\sigma_{\mathrm{SD}}$, because spin-independent scattering receives an $A^{2}$ enhancement from coherent scattering in the heavy nuclei of a detector. Most dark matter models will be probed first by $\sigma_{\mathrm{SI}}$-sensitive detectors, so it is worth considering which classes of models will probed first by $\sigma_{\mathrm{SD}}$-sensitive detectors.

An interesting class of such models are WIMPless dark matter models in which the dark matter candidate is a Majorana fermion. In these proceedings, we will study models of this type and consider detection prospects for IceCube/DeepCore.

\section{WIMPless Dark Matter}

WIMPless dark matter is a hidden sector particle with mass at the hidden sector soft SUSYbreaking scale [1]. The thermal relic density is set by the annihilation cross-section, $\rho \propto\left\langle\sigma_{\text {ann. }} v\right\rangle^{-1}$, which in turn is determined from dimensional analysis, $\left\langle\sigma_{a n n .} v\right\rangle \propto \frac{g^{4}}{m^{2}}$. To get the correct relic density, one must have $\left\langle\sigma_{a n n} . v\right\rangle \sim 1 \mathrm{pb}$. The "WIMP Miracle" is the remarkable coincidence that, for $g=g_{\text {weak }}$ and $m=m_{\text {weak }}$, the annihilation cross-section is indeed $\sim \mathrm{pb}$. In the WIMPless scenario, both the MSSM sector and the hidden sector receive the effects of SUSY-breaking through gauge-mediation from the same SUSY-breaking sector. The soft scale is thus set by the gaugecoupling, yielding the relation

$$
\frac{g^{2}}{m}, \frac{g_{\text {weak }}^{2}}{m_{\text {weak }}} \propto \frac{M_{\text {mess }}}{F}
$$

where $g$ and $m$ are the coupling and soft SUSY-breaking scale of the hidden sector, $F$ is the SUSYbreaking vev, and $M_{\text {mess. }}$ is the messenger mass scale. The WIMPless dark matter candidate thus naturally has approximately the same annihilation cross-section (and relic density) as a WIMP.

This is a very robust result, essentially determined by dimensional analysis and the powercounting of gauge interactions. The relic density calculation does not depend on whether the 
WIMPless candidate is a boson or a fermion. When the WIMPless candidate is a Majorana fermion, an interesting feature is that the dark matter-nucleon scattering cross-section may be largely spindependent. WIMPless dark matter can couple to Standard Model matter through Yukawa couplings

$$
V=\lambda_{L i} \tilde{Y}_{L} \bar{X} P_{L} f_{i}+\lambda_{R i} \tilde{Y}_{R} \bar{X} P_{R} f_{i}+\text { h.c. }
$$

where the $f_{i}$ are Standard Model fermions. Dark matter-nucleon scattering arises through coupling to Standard Model quarks, and in general WIMPless dark matter can couple to all generations.

The scattering cross-section can be determined from the Yukawa couplings above. The spindependent part of the scattering cross-section (assuming no squark mixing) is given by

$$
\sigma_{\mathrm{SD}}=\frac{m_{r}^{2}}{4 \pi} \frac{J+1}{J}\left[\sum_{i}\left(\frac{\left|\lambda_{L i}^{2}\right|}{m_{\tilde{Y}_{L}}^{2}-m_{X}^{2}}+\frac{\left|\lambda_{R i}^{2}\right|}{m_{\tilde{Y}_{R}}^{2}-m_{X}^{2}}\right)\left(\left\langle S_{p}\right\rangle \Delta_{i}^{(p)}+\left\langle S_{n}\right\rangle \Delta_{i}^{(n)}\right)\right]^{2},
$$

where

$$
\Delta_{u}^{(p)}=\Delta_{d}^{(n)}=0.78 \pm 0.02, \quad \Delta_{d}^{(p)}=\Delta_{u}^{(n)}=-0.48 \pm 0.02, \quad \Delta_{s}^{(p, n)}=-0.15 \pm 0.02 .
$$

are spin-structure functions. We will focus on the case where dark matter couples dominantly to the first generation quarks. If the WIMPless candidate is a Majorana fermion, then the only diagrams contributing to spin-independent scattering are those involving mixing of the 4th generation squarks, $\tilde{Y}_{L}$ and $\tilde{Y}_{R}$. The spin-independent scattering cross-section is thus proportional to $\operatorname{Re}\left(\lambda_{L} \lambda_{R}^{*}\right)^{2}$. If squark-mixing is small, or if $\lambda_{L} \ll \lambda_{R}$ (or $\lambda_{R} \ll \lambda_{L}$ ), the dark matter-nucleon scattering cross-section will be largely spin-dependent.

\section{Tests at IceCube/DeepCore}

The IceCube detector (with the DeepCore extension) is expected to soon have among the best sensitivity to $\sigma_{\mathrm{SD}}$ [5], obtained through bounds on the neutrino flux from dark matter which annihilates after being captured in the sun. Detection prospects at IceCube/DeepCore for such models were studied in [6], including the effects of neutrino propagation through the sun and vacuum. Detection prospects for the $\tau \bar{\tau}, \tilde{\tau} \tilde{\tau}^{*}$ and $\tilde{v} \tilde{v}^{*}$ annihilation channels are shown in Figure 1. In particular, it was found that $3 \sigma$-evidence of models with reasonable Yukawa couplings $\left(\lambda_{u, d} \sim\right.$ 0.5 ) could be found with 5 years of running time. As expected, the DeepCore extension is most important for low-energy neutrinos, resulting from either low-mass dark matter annihilation, or from annihilation to superpartners whose decay chain results in lower energy neutrinos. For higher mass dark matter, the best detection prospects arise from the events fully-contained within the IceCube volume.

\section{Conclusion}

WIMPless Majorana fermion models contain a class of well-motivated dark matter candidates for which IceCube/DeepCore may very well be a discovery experiment. The relative detection prospects at IceCube/DeepCore, as opposed to direct detection experiments such as CDMS or Xenon100, depend on the magnitude of 4th generation squark-mixing, as well as the relative strengths of the $\lambda_{L}$ and $\lambda_{R}$ Yukawa couplings. A detailed study of these cross-sections, and their effect on detection prospects, is currently underway [8]. 

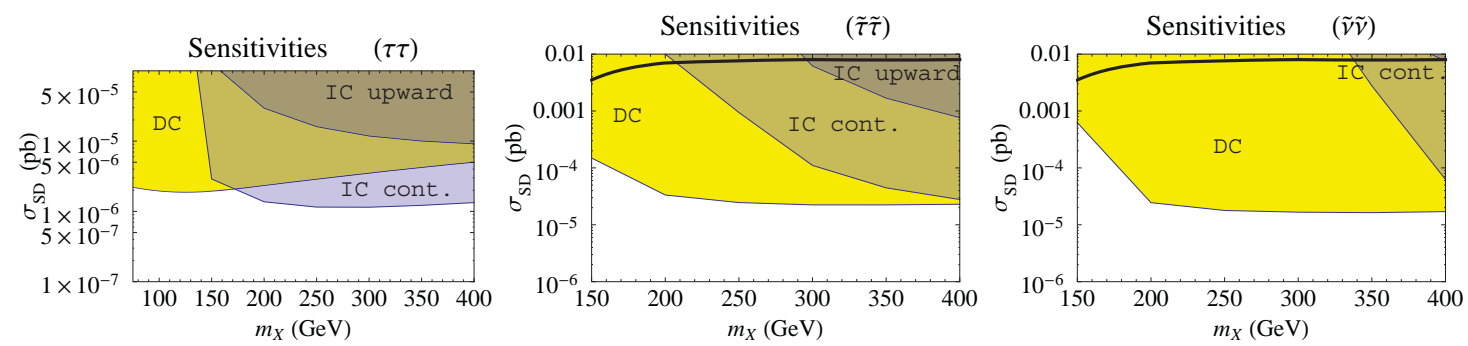

Figure 1: $3 \sigma$ detection prospects for Majorana fermion WIMPless dark matter annihilating exclusively to $\tau \bar{\tau}$ (left), $\tilde{\tau} \tilde{\tau}^{*}$ (middle) and $\tilde{v} \tilde{v}^{*}$ (right). We assume $m_{\tilde{\tau}_{1}}=137 \mathrm{GeV}, m_{\tilde{v}}=111.5 \mathrm{GeV}, m_{\tilde{\chi}_{1}^{0}}=94.5 \mathrm{GeV}$, and the decays $\tilde{\tau} \rightarrow \tau \tilde{\chi}_{1}^{0}, \tilde{v} \rightarrow v \tilde{\chi}_{1}^{0}$. We assume that the sneutrino Yukawa couplings are flavor-independent. The dark grey region (IC upward), blue region (IC contained), and the light brown region (DC) indicate detection prospects using the upward throughgoing sample from IceCube, the muon sample fully contained within the IceCube volume, and muon sample fully contained within the DeepCore volume, respectively. The solid black line is the bound from Super-Kamiokande [7]. (Figure courtesy of Danny Marfatia and Enrico Sessolo.)

\section{Acknowledgments}

We are grateful to the organizers of ICHEP2010, and to V. Barger, D. Marfatia, and E. Sessolo for collaboration and J. L. Feng, K. Fukushima and P. Sandick for discussions. JK is supported by DOE grant DE-FG02-04ER41291.

\section{References}

[1] J. L. Feng and J. Kumar, Phys. Rev. Lett. 101, 231301 (2008) [arXiv:0803.4196 [hep-ph]].

[2] J. L. Feng, J. Kumar and L. E. Strigari, Phys. Lett. B 670, 37 (2008) [arXiv:0806.3746 [hep-ph]].

[3] R. Bernabei et al., Eur. Phys. J. C 67, 39 (2010) [arXiv:1002.1028 [astro-ph.GA]]; C. E. Aalseth et al. [CoGeNT collaboration], arXiv:1002.4703 [astro-ph.CO]; D. Hooper, J. I. Collar, J. Hall and D. McKinsey, arXiv:1007.1005 [hep-ph]; see talk of W. Seidel at IDM2010, http://indico.in2p3.fr/contributionDisplay.py? contribId=195\&session $I d=9 \& \operatorname{conf} I d=1565$

[4] D. Hooper, F. Petriello, K. M. Zurek and M. Kamionkowski, Phys. Rev. D 79, 015010 (2009) [arXiv:0808.2464 [hep-ph]]; J. L. Feng, J. Kumar, J. Learned and L. E. Strigari, JCAP 0901, 032 (2009) [arXiv:0808.4151 [hep-ph]]; J. Kumar, J. G. Learned and S. Smith, Phys. Rev. D 80, 113002 (2009) [arXiv:0908.1768 [hep-ph]]; J. Alwall, J. L. Feng, J. Kumar and S. Su, Phys. Rev. D 81, 114027 (2010) [arXiv:1002.3366 [hep-ph]].

[5] J. Braun and D. Hubert for the IceCube Collaboration, arXiv:0906.1615 [astro-ph.HE].

[6] V. Barger, J. Kumar, D. Marfatia and E. M. Sessolo, Phys. Rev. D 81, 115010 (2010) [arXiv:1004.4573 [hep-ph]].

[7] S. Desai et al. [Super-Kamiokande Collaboration], Phys. Rev. D 70, 083523 (2004) [Erratum-ibid. D 70, 109901 (2004)] [arXiv:hep-ex/0404025].

[8] J. L. Feng, K. Fukushima, J. Kumar and P. Sandick, work in progress. 